\title{
Motion Control of Four-Wheel Independently Actuated Electric Ground Vehicles considering Tire Force Saturations
}

\author{
Rongrong Wang, ${ }^{1}$ Hamid Reza Karimi, ${ }^{2}$ Nan Chen, ${ }^{1}$ Guodong Yin, ${ }^{1}$ and Jinxiang Wang ${ }^{1}$ \\ ${ }^{1}$ School of Mechanical Engineering, Southeast University, Nanjing 211189, China \\ ${ }^{2}$ Department of Engineering, Faculty of Engineering and Science, University of Agder, 4898 Grimstad, Norway \\ Correspondence should be addressed to Rongrong Wang; wrr06fy@gmail.com
}

Received 26 September 2013; Accepted 17 November 2013

Academic Editor: Hui Zhang

Copyright (c) 2013 Rongrong Wang et al. This is an open access article distributed under the Creative Commons Attribution License, which permits unrestricted use, distribution, and reproduction in any medium, provided the original work is properly cited.

\begin{abstract}
A vehicle stability control approach for four-wheel independently actuated (FWIA) electric vehicles is presented. The proposed control method consists of a higher-level controller and a lower-level controller. An adaptive control-based higher-level controller is designed to yield the vehicle virtual control efforts to track the desired vehicle motions due to the possible modeling inaccuracies and parametric uncertainties. The lower-level controller considering tire force saturation is given to allocate the required control efforts to the four in-wheel motors for providing the desired tire forces. An analytic method is given to distribute the high-level control efforts, without using the numerical-optimization-based control allocation algorithms. Simulations based on a high-fidelity, CarSim, and full-vehicle model show the effectiveness of the control approach.
\end{abstract}

\section{Introduction}

Electric vehicles typically achieve greater fuel economy, lower emissions, and increased energy security than conventional internal combustion engine vehicles [1]. Four-wheel independently actuated (FWIA) electric vehicles employ four inwheel (or hub) motors to actuate the four wheels, and thus the torque of each wheel can be controlled independently. The actuation flexibility of the FWIA electric vehicles together with the fast and precise torque responses of electric motors enhances the vehicle control strategies, such as the traction control system and direct yaw-moment control [2-4].

The actuators in a FWIA electric vehicle are more than those in a conventional vehicle. This actuator redundancy makes the FWIA electric vehicle control problem more challenging but rewarding. This paper considers the motion control problems of FWIA electric vehicles. Both the vehicle longitudinal speed and yaw motion are controlled. Many studies have been carried out on the vehicle control methods for improving the vehicle stability and maneuverability. Most of them nevertheless are designed for the conventional vehicle architectures [5-7], not for the FWIA electric vehicles. Sakai et al. [8] proposed a direct yaw-moment control system for a FWIA electric vehicle, a half-vehicle model which is a linear approximation of vehicle dynamics was used in the controller design, and only the vehicle lateral motion was controlled. A braking control method for electric vehicle was proposed in [9], and the studied vehicle was driven by independent front and rear motors. The vehicle stability problem was not considered either in the paper. A stability control method for four-wheel driven hybrid electric vehicle was proposed in [10]. The studied vehicle in this paper was driven by a front and a rear motor, and the rear motor with an electrohydraulic brake was used to generate the required torque split for yaw motion control. As a FWIA electric vehicle is equipped with four in-wheel motors to independently actuate the four wheels, the control problem in [10] is thus different from the one considered in this study.

In this paper, the tracking control problem [11-14] of a FWIA electric vehicle is studied. The proposed control system consists of a higher-level controller and a lowerlevel controller. Due to the possible modeling inaccuracies and vehicle parametric uncertainties, an adaptive controller is designed as the higher-level control to give the required virtual total ground forces and the force split between the left and right sides of the vehicle. The vehicle longitudinal 


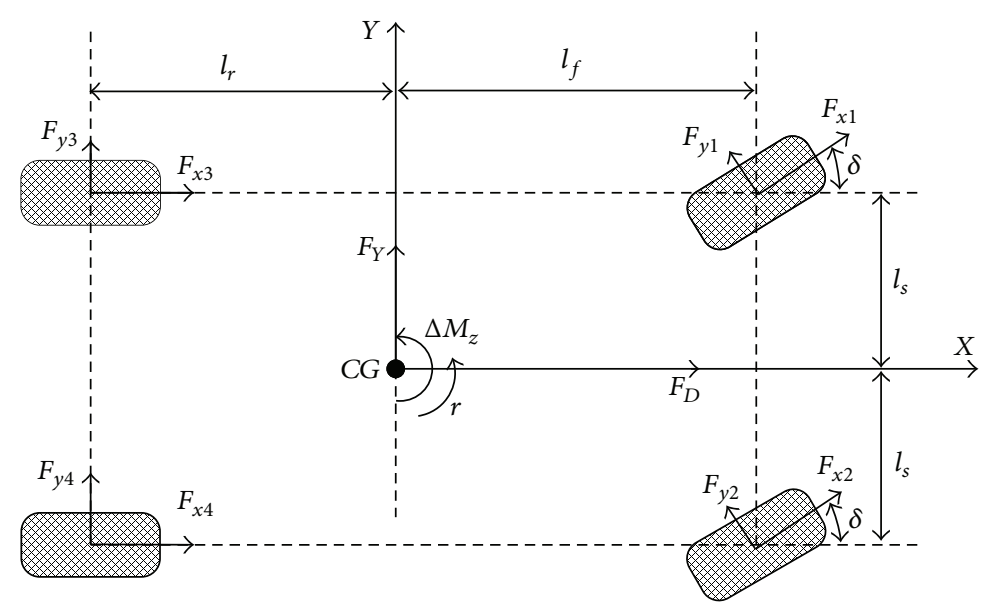

FIGURE 1: Schematic diagram of a vehicle planar motion model.

speed is controlled by the total ground forces while the yaw motion is compensated with the external yaw moment generated with the tire force difference between the two sides of the vehicle. The lower-level controller allocates the virtual ground forces from the higher-level controller to the four wheels. Control allocation algorithms are generally used to distribute the higher-level control signals to the lower-level actuators $[15,16]$. As the control allocation algorithms usually require high computational costs, which may discourage their implementations in real time, an analytic solution of allocating the ground forces without using the numericaloptimization-based control allocation algorithms is given in this study. When the tire slip ratios become large and move into the unstable tire force region, the tire forces will be saturated and it may no longer be possible to fully transfer the desired tire force onto the road. What is more, locking/skidding wheels no longer provide any grip on the road and thus the cornering forces transferred to the ground will be limited. So the vehicle will be unsteerable if the tire slip ratios become too large. The constraints of the tire forces are also explicitly considered in the optimal solution.

The rest of the paper is organized as follows. System modeling is presented in Section 2. The proposed higher-level controllers are designed in Section 3. Tire force distribution design considering the tire force constraints is described in Section 4. Simulation results based on a high-fidelity, CarSim, full-vehicle model are provided in Section 5 followed by conclusive remarks.

\section{System Modelling}

Vehicle yaw control enhances the vehicle handling performance and maintains vehicle stability in cornering maneuvers [17]. When the vehicle yaw rate can be controlled to track the reference, the vehicle lateral speed and slip angle will be small [18]. A schematic diagram of a vehicle model is shown in Figure 1. If the vehicle longitudinal speed and yaw rate are controlled at the same time, the vehicle model can be expressed as

$$
\begin{aligned}
\dot{v}_{x}=v_{y} r-\frac{C_{a}}{m} v_{x}^{2}+\frac{1}{m} \sum_{i=1}^{2} F_{y i} \sin (\delta)+\frac{F_{D}}{m} \\
\dot{r}=\frac{1}{I_{z}}\left(\sum_{i=1}^{2} F_{y i}\left(l_{f} \cos (\delta)+(-1)^{i} l_{s} \sin (\delta)\right)-\sum_{i=3}^{4} l_{r} F_{y i}\right) \\
+\frac{1}{I_{z}} \Delta M_{z},
\end{aligned}
$$

where $v_{x}$ and $v_{y}$ are the vehicle longitudinal speed and lateral speed, respectively, $r$ is the yaw rate, $m$ is the mass of the vehicle, $I_{z}$ is the yaw inertia, and $C_{a}$ is the aerodynamic drag term. $F_{D}$ is the total driving/braking forces in vehicle longitudinal direction, $\Delta M_{z}$ is the external yaw moment generated with the longitudinal tire force difference between the left and right side wheels, $F_{D}$ and $\Delta M_{z}$ can be written as

$$
\begin{gathered}
F_{D}=\sum_{i=1}^{2} F_{x i} \cos (\delta)+\sum_{3=1}^{4} F_{x i}, \\
\Delta M_{z}=\sum_{i=1}^{2} F_{x i}\left((-1)^{i} \cos (\delta) l_{s}+\sin (\delta) l_{f}\right) \\
+\sum_{i=3}^{4}(-1)^{i} l_{s} F_{x i} .
\end{gathered}
$$

The wheel slip angle is a function of the vehicle states and can be calculated as

$$
\begin{aligned}
& \beta_{1,2}=-\delta+\tan ^{-1}\left(\frac{v_{y}+l_{f} r}{v_{x} \mp l_{s} r}\right), \\
& \beta_{3,4}=\tan ^{-1}\left(\frac{v_{y}-l_{r} r}{v_{x} \mp l_{s} r}\right) .
\end{aligned}
$$


The tire lateral forces are functions of the tire slip angles which can be calculated with the vehicle states, which indicates that the tire lateral forces in $F_{y i}$ are also functions of the vehicle states. Denoting $x=\left[\begin{array}{lll}v_{x} & v_{y} & r\end{array}\right]^{T}$, the vehicle model can be rewritten as

$$
\begin{aligned}
& \dot{x}_{1}=f_{1}(x)+\frac{1}{m} u_{1}, \\
& \dot{x}_{2}=f_{2}(x)+\frac{1}{I_{z}} u_{2},
\end{aligned}
$$

where $u_{1}=F_{D}$ and $u_{2}=\Delta M_{z}$ are the control signals, $f_{1}(x)=v_{y} r-\left(C_{a} / m\right) v_{x}^{2}+(1 / m) \sum_{i=1}^{2} F_{y i} \sin (\delta)$, and $f_{2}(x)=$ $\left(1 / I_{z}\right)\left(\sum_{i=1}^{2} F_{y i}\left(l_{f} \cos (\delta)+(-1)^{i} l_{s} \sin (\delta)\right)-\sum_{i=3}^{4} l_{r} F_{y i}\right)$.

The tire longitudinal slip ratio $s_{i}$ is defined as the relative difference between the tire center speed and tire circumferential speed, and the tire slip ratio can be written as

$$
s_{i}=\frac{\omega_{i} R-v_{x i}}{\max \left(v_{x i}, \omega_{i} R\right)},
$$

where $\omega_{i}$ is the tire longitudinal rotational speed of the $i$ th wheel, $R$ is the tire effective rolling radius, and $v_{x i}$ are the speeds at the centers of the wheels and are given as

$$
\begin{aligned}
& v_{x 1,2}=\left(v_{x} \mp l_{s} r\right) \cos \delta+\left(v_{y}+l_{f} r\right) \sin \delta, \\
& v_{x 3,4}=v_{x} \mp l_{s} r .
\end{aligned}
$$

The vehicle states can be measured with the global positioning system (GPS) and inertia measurement unit (IMU) $[19,20]$. The wheel speeds can be measured with wheel speed sensors. Thus, in this study, we assume all of the required signals to be known.

\section{Higher-Level Controller Design}

The proposed control system consists of a higher-level controller and a lower-level controller. The vehicle longitudinal speed is controlled by the total ground forces while the yaw rate is compensated with the external yaw moment. For the first channel of (4), the following controller

$$
u_{1}^{*}=m\left(-f_{1}(x)+K_{1} e_{x}+\dot{v}_{x r}\right),
$$

with $v_{x r}$ being the reference vehicle longitudinal speed and $K_{1}$ being a positive constant, can make the longitudinal speed tracking error, $e_{x}=v_{x r}-v_{x}$, converge to 0. However, $f_{1}(x)$ and the vehicle mass $m$ may not be accurately obtained due to the modeling error and parameter uncertainties, and an adaptive controller is thus designed to yield the control signal $u_{1}$. The controller for the first channel is thus modified as

$$
u_{1}=\widehat{m}\left(-\widehat{f}_{1}(x)+K_{1} e_{x}+\dot{v}_{x r}\right),
$$

where $\widehat{f}_{1}$ and $\widehat{m}$ are the estimated values of $f_{1}(x)$ and $m$, respectively. If the following control law are used:

$$
\dot{\hat{f}}_{1}=-\gamma_{1} e_{x}, \quad \dot{\vec{m}}=e_{x} \gamma_{2} u_{1} \text {, }
$$

with $\gamma_{1}$ and $\gamma_{2}$ being positive constants, the longitudinal speed tracking error $e_{x}$ can be bounded as

$$
\left|e_{x}\right|=\sqrt{\frac{\left(f_{1 \max }-f_{1 \min }\right)\left|\dot{f}_{1}\right|_{\max }}{K_{1} \gamma_{1}}},
$$

where $\left|\dot{f}_{1}\right|_{\max }$ is the upper boundary of $\left|\dot{f}_{1}\right|$ and $f_{1 \max }$ and $f_{1 \mathrm{~min}}$ are the upper and lower boundaries of $f_{1}(x)$, respectively. Proof can be found in Appendix A. Note that by choosing sufficiently large $\gamma_{1}$ and $K_{1}$, the longitudinal speed tracking error $e_{x}$ can be arbitrarily small.

The adaption law for $\widehat{f}_{1}$ and $\widehat{m}$ in (9) may cause the control signals grow out of the boundary. Thus the following control law modifications are introduced:

$$
\begin{aligned}
& u_{1}=\widehat{m}\left(-\widehat{f}_{1}(x)+K_{1} e_{x}+\dot{v}_{x r}\right), \\
& \dot{\bar{f}}=-\gamma_{1} e_{x}-\kappa_{1}\left(\bar{f}_{1}-\widehat{f}_{1}\right), \\
& \dot{\bar{m}}=e_{x} \gamma_{2} u_{1}-\kappa_{2}(\bar{m}-\widehat{m}),
\end{aligned}
$$

where $\kappa_{1}$ and $\kappa_{2}$ are positive constants,

$$
\begin{gathered}
\widehat{f}_{1}= \begin{cases}\bar{f}_{1} & \text { if } f_{1 \min } \leq \bar{f}_{1} \leq f_{1 \max } \\
f_{1 \min } & \text { if } \bar{f}_{1}<f_{1 \min } \\
f_{1 \max } & \text { if } \bar{f}_{1}>f_{1 \max },\end{cases} \\
\widehat{m}= \begin{cases}\bar{m} & \text { if } m_{\min } \leq \bar{m} \leq m_{\max } \\
m_{\min } & \text { if } \bar{m}<m_{\min } \\
m_{\max } & \text { if } \bar{m}>m_{\max },\end{cases}
\end{gathered}
$$

with $m_{\max }$ and $m_{\min }$ being the upper and lower bounds of $m$. The modified control law (11) for the first channel can still make the tracking error $e_{x}$ be arbitrarily small. Proof can be found in Appendix B.

Similar to the controller design for the first channel, the control law for the second channel can be designed as

$$
\begin{gathered}
u_{2}=\frac{\widehat{I}_{z}}{l_{s}}\left(-\widehat{f}_{2}+K_{2} e_{2}+\dot{r}_{\text {ref }}\right), \\
\dot{\bar{f}}_{2}=-\gamma_{3} e_{x}-\kappa_{3}\left(\bar{f}_{2}-\widehat{f}_{2}\right), \\
\dot{\bar{I}}_{z}=e_{2} \gamma_{4} l_{s} u_{2}-\kappa_{4}(\bar{m}-\widehat{m}),
\end{gathered}
$$

where $e_{2}=r_{\text {ref }}-r$ is the yaw rate tracking error and $\gamma_{3}, \gamma_{4}, \kappa_{3}$, and $\kappa_{4}$ are positive constants,

$$
\begin{aligned}
& \hat{f}_{2}= \begin{cases}\bar{f}_{2} & \text { if } f_{1 \min } \leq \bar{f}_{2} \leq f_{2 \max } \\
f_{2 \min } & \text { if } \bar{f}_{2}<f_{2 \min } \\
f_{2 \max } & \text { if } \bar{f}_{2}>f_{2 \max },\end{cases} \\
& \widehat{I}_{z}= \begin{cases}\bar{I}_{z} & \text { if } I_{z \min } \leq \bar{I}_{z} \leq I_{z \max } \\
I_{z \min } & \text { if } \bar{I}_{z}<I_{z \min } \\
I_{z \max } & \text { if } \bar{I}_{z}>I_{z \max } .\end{cases}
\end{aligned}
$$


Here, $f_{2 \max }$ and $f_{2 \min }$ are the upper and lower bounds of $f_{2}$, respectively. $I_{z \max }$ and $I_{z \text { min }}$ are the upper and lower bounds of $I_{z}$, respectively.

\section{Lower-Level Controller Design}

When the higher-level controller signals are obtained, the lower-level controller operates the four in-wheel motors such that the control requirements from the higher-level controller can be satisfied. An analytic solution is given to distribute the higher-level control efforts without using the numericaloptimization-based control allocation algorithms.

The cost function for allocating the four tire forces can be defined as

$$
J=\frac{1}{2}\left(F_{x}^{T} W F_{x}+\left(B F_{x}-u\right)^{T} Q\left(B F_{x}-u\right)\right),
$$

where $Q=\operatorname{diag}\left[\begin{array}{ll}q_{1} & q_{2}\end{array}\right], W=\operatorname{diag}\left[\begin{array}{llll}w_{1} & w_{2} & w_{3} & w_{4}\end{array}\right], F_{x}=$ $\left[\begin{array}{llll}F_{x 1} & F_{x 2} & F_{x 3} & F_{x 4}\end{array}\right]^{T}, u=\left[u_{1}, u_{2}\right]^{T}$ is the desired control effort given by the higher-level controller, and $B$ is the control effectiveness matrix and can be written according to (2)

$$
B=\left[\begin{array}{cccc}
\cos (\delta) & \cos (\delta) & 1 & 1 \\
-\cos (\delta) l_{s}+\sin (\delta) l_{f} & \cos (\delta) l_{s}+\sin (\delta) l_{f} & -l_{s} & l_{s}
\end{array}\right] .
$$

Based on (15), we have

$$
\begin{aligned}
\frac{\partial J}{\partial F_{x}} & =F_{x}^{T} W+F_{x}^{T} B^{T} Q B-B^{T} Q u \\
& =F_{x}^{T}\left(W+B^{T} Q B\right)-B^{T} Q u \\
H & =\frac{\partial^{2} J}{\partial^{2} F_{x}}=W+B^{T} Q B .
\end{aligned}
$$

As $W>0$ and $B^{T} Q B \geq 0$, one can claim $H>0$, which implies that the objective function $J$ has a global minimum with the minimizing $F_{x 0}$ given by

$$
F_{x 0}=\left(W+B^{T} Q B\right)^{-1} B^{T} Q u .
$$

It is known that the tire force may become saturated if a sufficiently large motor control signal is applied in some extreme cases such as hard brake on a low- $\mu$ road. Once the tire longitudinal force reaches its maximal value, further increasing of slip makes the tire work in the unstable range and the tire force will decrease quickly. So the constraints of the tire forces should be explicitly considered in the tire force allocation design. Note that a bigger weighting factor $w_{i}$ in (15) for a wheel means that a smaller portion of the total torque is required from this wheel. So one can control a certain wheel to provide a larger or less portion of the total torque by selecting a smaller or bigger weighting factor for this wheel. The constraint violations of the tire forces can be discouraged by defining the weighting factors $w_{i}$ in (15) as

$$
w_{i}=\frac{w_{0}}{\left(1-s_{i}^{*}\right)^{\kappa}},
$$

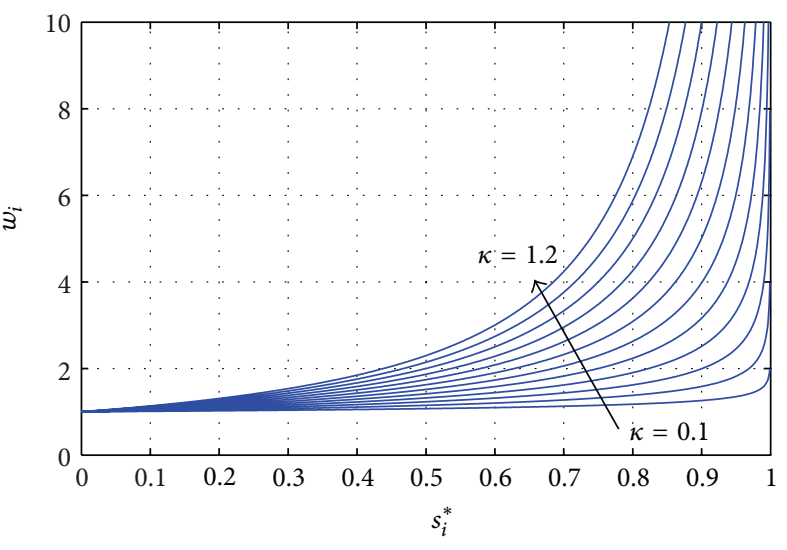

FIGURE 2: Weighting factor curves at different $\kappa\left(w_{0}=1\right)$.

where $w_{0}$ is a positive constant, $\kappa>0$ is a constant which is used to determine the shape of the weighting factor, and $s_{i}^{*}=s_{i} / s_{i}^{\text {peak }}$ is the normalized tire slip ratio with $s_{i}^{\text {peak }}$ corresponding to the maximum longitudinal forces of the $i$ th tire. The curves of the weighting factor at different $\kappa>$ 0 are plotted in Figure 2. When the slip ratio of a certain wheel reaches the $s_{i}^{\text {peak }}$ which corresponds to the maximum longitudinal tire force, the weighting factor for this wheel will become large according to (20), which means that the required tire force from this wheel will become small; in this way the tire slip ratio can always be limited within the stable region. Based on the desired tire force calculated from (19), motor control signals can be generated such that the desired tire forces can be provided [21].

\section{Simulation Results}

Two simulation cases based on a high-fidelity, full-vehicle model constructed in CarSim were conducted. The vehicle parameters in the simulations were taken from an actual FWIA electric vehicle with in-wheel motors developed at the Ohio State University [21]. The desired vehicle yaw rate and speed can be generated from the drivers steering angle, accelerator/brake pedal positions. The vehicle reference model can be found in the literatures such as $[22,23]$.

5.1. J-Turn Simulation. In this simulation, the vehicle ran at a low-speed range. A counter-clockwise turn was introduced with the front wheel steering angle shown in Figure 3. We aim at controlling the vehicle such that the actual vehicle states can follow the references. The nominal vehicle mass was set to $800 \mathrm{~kg}$ in the simulation. As the designed controller does not depend on the actual vehicle parameters, we set the vehicle mass in the controller as $600 \mathrm{~kg}$, which is different than the actual vehicle mass.

The generated external yaw moment with the tire force difference between two sides of the vehicle is shown in Figure 4 . The vehicle yaw rate and vehicle speed are plotted in Figures 5 and 6, respectively. One can see from these two figures that both vehicle yaw rate and speed could be well 


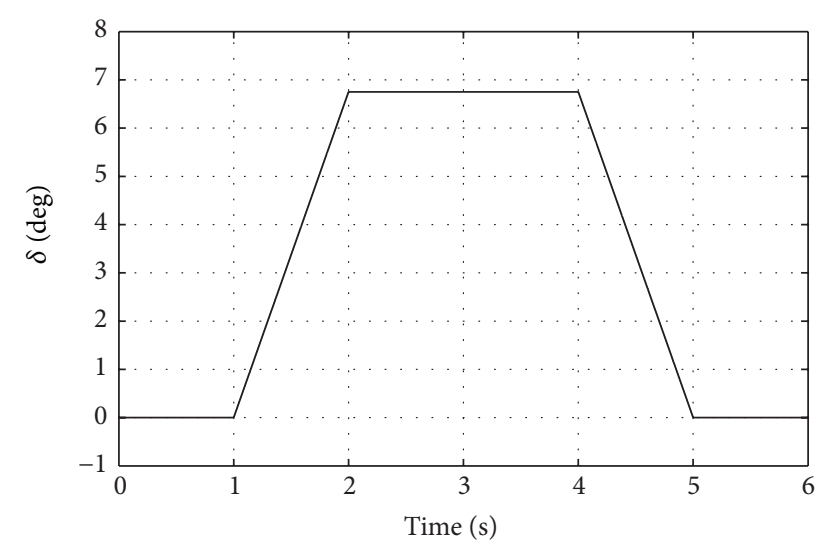

FIGURE 3: Front wheel steering angle in the J-turn simulation.

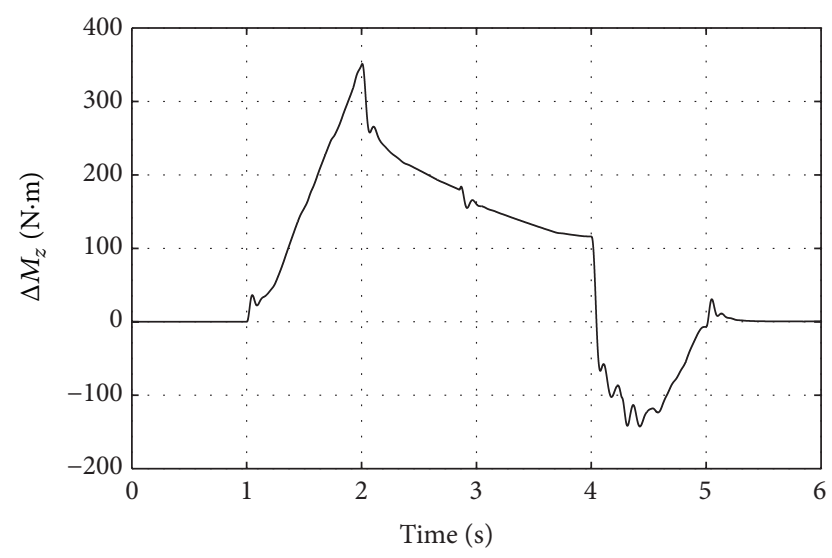

Figure 4: The generated external yaw moment in the J-turn simulation.

controlled. Note that, in this simulation, a big steering angle was applied to the vehicle, and this steering angle could make the vehicle yaw rate reach up to $30 \mathrm{deg} / \mathrm{s}$, which indicates that the proposed control method can control the vehicle well at extreme conditions.

5.2. Single-Lane Change. In the above simulation, we investigated the performance of the proposed controller in the cases where the vehicle runs on a high- $\mu$ road. In this simulation, the vehicle was controlled to make a single-lane change on a low- $\mu$ road. The tire-road friction coefficient was set as 0.2 and a big steering which would make the vehicle loss of stability was introduced at $2 \mathrm{~s}$. The desired speed increased from $24.5 \mathrm{~m} / \mathrm{s}$ to around $27.8 \mathrm{~m} / \mathrm{s}$ in 6 seconds. The vehicle mass in the controller was set to $1000 \mathrm{~kg}$, which is bigger than the actual vehicle mass.

The front wheel steering angle is shown in Figure 7. The generated yaw moment which regulated the vehicle yaw rate was plotted in Figure 8. The yaw rate control results are shown in Figure 9. To better show the effectiveness of the proposed control method, the yaw rate of an uncontrolled vehicle which ran on the same low- $\mu$ road was compared. One can see from Figure 9 that the yaw rate of the controlled

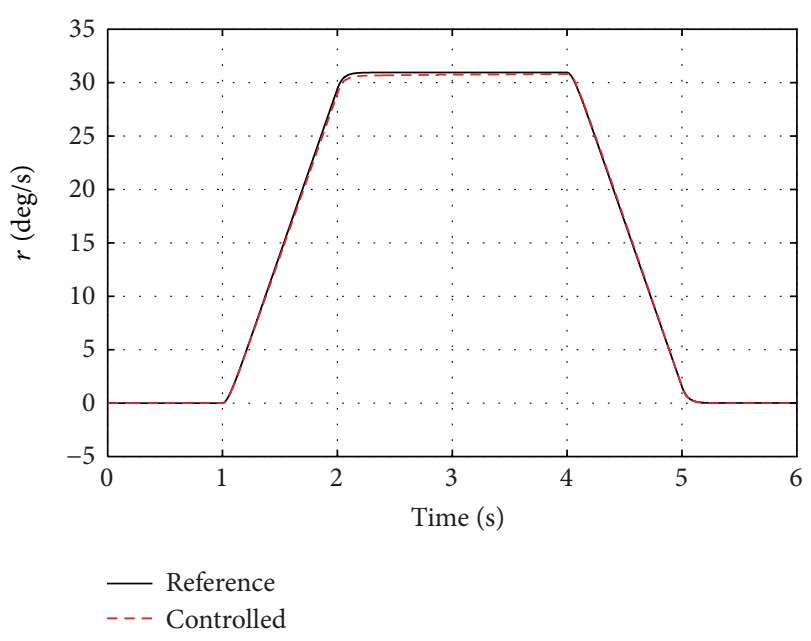

FIGURE 5: Vehicle yaw rates in the J-turn simulation.

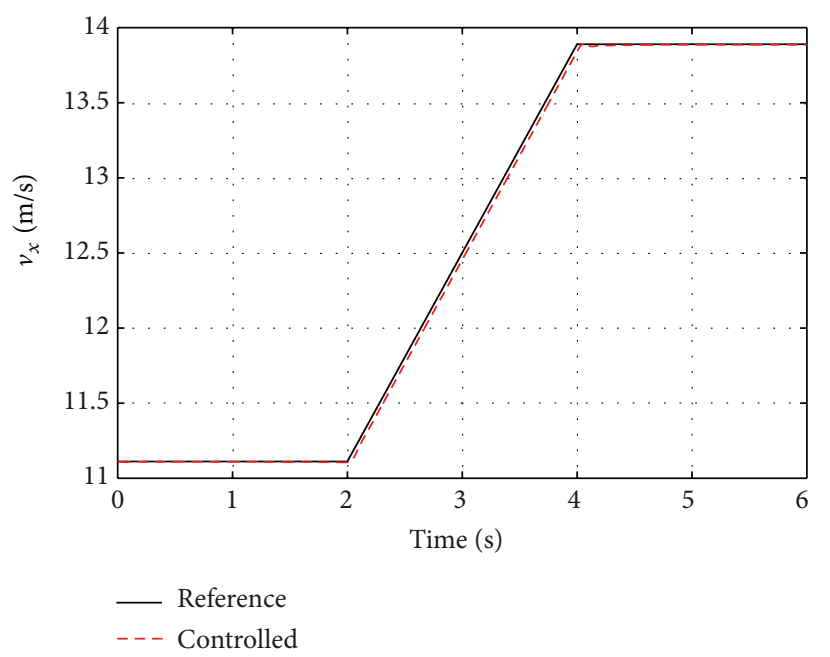

Figure 6: Vehicle speeds in the J-turn simulation.

vehicle could always follow the reference, while the yaw rate of the uncontrolled vehicle deviated from the reference when the front wheel steering angle became large. The yaw rate control results indicate that stability of the controlled vehicle was ensured. The vehicle longitudinal speeds are plotted in Figure 10. One can see again that the vehicle speed could be well controlled as well.

\section{Conclusion}

A vehicle stability control system for an FWIA electric vehicle is presented. The proposed adaptive control-based higherlevel controller does not need the accurate vehicle parameters or tire force models but can still yield the desired control signals. An analytic solution considering tire force constraints is designed to allocate the required control efforts from the higher-lever controller to the four wheels. Simulations under various driving scenarios are carried out with a high-fidelity, 


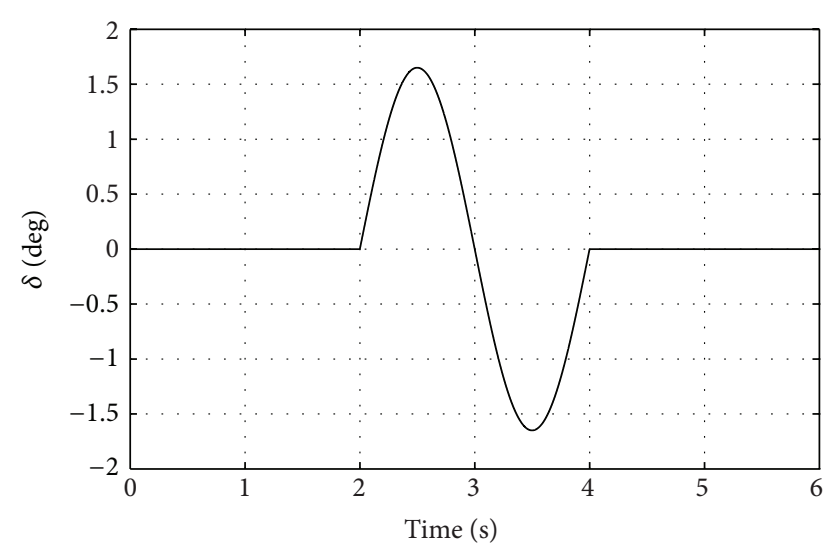

FIgURE 7: Front wheel steering angle in the single-lane change simulation.

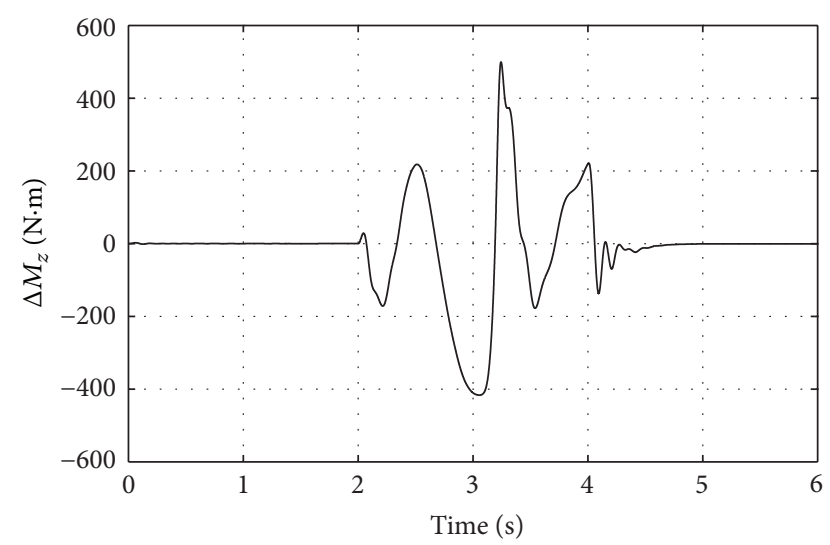

Figure 8: The generated external yaw moment in the single-lane change simulation.

CarSim, and full-vehicle model. Simulation results show the effectiveness of the proposed control approach.

\section{Appendices}

A.

By defining $\widetilde{m}=\widehat{m}-m$ and $\widetilde{f}_{1}=\widehat{f}_{1}-f_{1}$, the dynamics of the vehicle speed can be written as

$$
\begin{aligned}
\dot{v}_{x} & =f_{1}+\frac{1}{m} u_{1} \\
& =\left(\widehat{f}_{1}-\widetilde{f}_{1}\right)+\frac{1}{m}(m+\widetilde{m})\left(-\widehat{f}_{1}+K_{1} e_{1} \dot{v}_{r x}\right) \\
& =\left(\left(\widehat{f}_{1}-\widetilde{f}_{1}\right)+\left(-\widehat{f}_{1}+K_{1} e_{1}+\dot{v}_{r x}\right)\right)+\frac{\widetilde{m}}{m} u_{1} \\
& =\left(-\widetilde{f}_{1}+K_{1} e_{1}+\dot{v}_{r x}\right)+\frac{\widetilde{m}}{m} u_{1},
\end{aligned}
$$

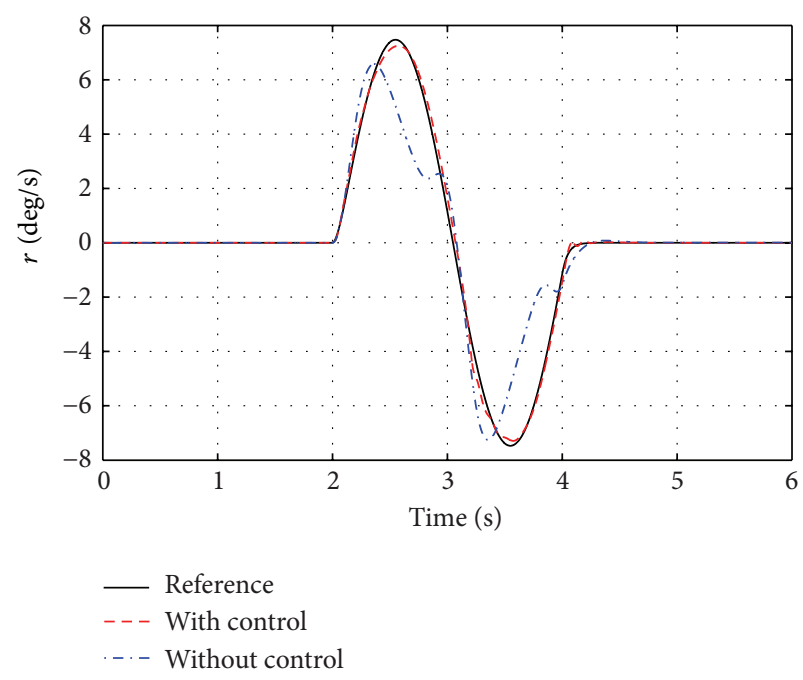

Figure 9: Vehicle yaw rates in the single-lane change simulation.

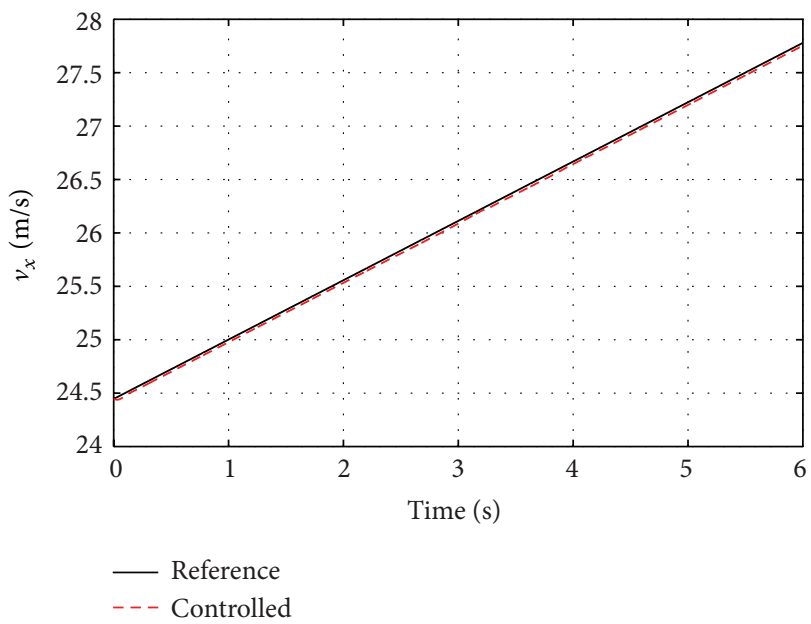

FIGURE 10: Vehicle longitudinal speeds in the single-lane change simulation.

which means that the error dynamics of the first channel can be written as

$$
\begin{aligned}
\dot{e}_{1} & =\dot{v}_{r x}-\dot{v}_{x} \\
& =-K_{1} e_{1}+\widetilde{f}_{1}-\frac{\widetilde{m}}{m} u_{1} .
\end{aligned}
$$

Define the Lyapunov function candidate for this channel as

$$
V_{1}=\frac{1}{2}\left(e_{1}^{2}+\frac{\tilde{f}_{1}^{2}}{\gamma_{1}}+\frac{\widetilde{m}^{2}}{\gamma_{2} m}\right)
$$


whose time derivative can be expressed as

$$
\begin{aligned}
\dot{V}_{1} & =e_{1} \dot{e}_{1}-\frac{\tilde{f}_{1}\left(\dot{\hat{f}}_{1}-\dot{f}_{1}\right)}{\gamma_{1}} \dot{+} \frac{\tilde{m} \dot{\hat{m}}}{\gamma_{2} m} \\
& =e_{1}\left(-K_{1} e_{1}+\widetilde{f}_{1}-\frac{\widetilde{m}}{m} u_{1}\right)-\frac{\tilde{f}_{1}\left(\dot{\hat{f}}_{1}-\dot{f}_{1}\right)}{\gamma_{1}}+\frac{\widetilde{m} \dot{\hat{m}}}{\gamma_{2} m} \\
& =-K_{1} e_{1}^{2}+\left(e_{x} \tilde{f}_{1}+\frac{\widetilde{f}_{1} \dot{\hat{f}}_{1}}{\gamma_{1}}\right)-\left(\frac{e_{x} \widetilde{m} u_{1}}{m}-\frac{\widetilde{m} \dot{\vec{m}}}{\gamma_{2} m}\right)-\frac{\tilde{f}_{1} \dot{f}_{1}}{\gamma_{1}} .
\end{aligned}
$$

Assuming that the upper bound of $\widehat{f}_{1}$ is the same as that for $f_{1}$, the update law (11) can make the time derivative of $V_{1}$ to be written as

$$
\begin{aligned}
\dot{V}_{1} & =-K_{1} e_{1}^{2}-\frac{\widetilde{f}_{1} \dot{f}_{1}}{\gamma_{1}} \\
& \leq-K_{1} e_{1}^{2}-\frac{\left|\widehat{f}_{1}-f_{1}\right|\left|\dot{f}_{1}\right|}{\gamma_{1}} \\
& \leq-K_{1} e_{1}^{2}-\frac{\left(\widehat{f}_{1 \max }-f_{1 \min }\right)\left|\dot{f}_{1}\right|_{\max }}{\gamma_{1}},
\end{aligned}
$$

where $\left|\dot{f}_{1}\right|_{\max }$ is the upper boundary of $\left|\dot{f}_{1}\right|$ and $f_{1 \text { min }}$ and $f_{1 \max }$ are the upper and lower boundaries of $f_{1}$, respectively. The error can be bounded according to (10). The proof is completed.

B.

Redefine the Lyapunov function candidate (A.3) as

$$
\begin{array}{r}
V_{1}=\frac{1}{2}\left(e_{1}^{2}+\frac{\left(\bar{f}_{1}-f_{1}\right)^{2}-\left(\bar{f}_{1}-\widehat{f}_{1}\right)^{2}}{\gamma_{1}}\right. \\
\left.+\frac{(\bar{m}-m)^{2}-(\bar{m}-\widehat{m})^{2}}{m \gamma_{2}}\right) .
\end{array}
$$

Based on (12), the time derivative of the above Lyapunov function candidate is

$$
\begin{aligned}
\dot{V}_{1}= & e_{1}\left(-K_{1} e_{1}+\tilde{f}_{1}-\frac{\widetilde{m}}{m} u_{1}\right) \\
& -\frac{\left(\tilde{f}_{1}-f_{1}\right)\left(\dot{\tilde{f}}_{1}-\dot{f}_{1}\right)-\left(\tilde{f}_{1}-\widehat{f}_{1}\right)\left(\dot{\tilde{f}}_{1}-\dot{\hat{f}}_{1}\right)}{\gamma_{1}} \\
& -\frac{(\widetilde{m}-m)(\dot{\vec{m}}-\dot{m})-(\widetilde{m}-\widehat{m})(\dot{\vec{m}}-\dot{\vec{m}})}{\gamma_{2} m}
\end{aligned}
$$

$$
\begin{aligned}
= & -K_{1} e_{1}^{2}+\left(\left(\gamma_{1} e_{1}\left(\hat{f}_{1}-f_{1}\right)-\bar{f}_{1} \dot{f}_{1}-f_{1} \dot{\bar{f}}_{1}+f_{1} \dot{f}_{1}\right.\right. \\
& \left.\left.+\bar{f}_{1} \dot{\hat{f}}_{1}+\widehat{f}_{1} \dot{\bar{f}}_{1}-\widehat{f}_{1} \dot{\hat{f}}_{1}\right) \times\left(\gamma_{1}\right)^{-1}\right) \\
& +\frac{\gamma_{2} e_{2}(\widehat{m}-m) u_{1}-m \dot{\bar{m}}-\widehat{m} \dot{\bar{m}}+\bar{m} \dot{\bar{m}}-\widehat{m} \dot{\bar{m}}}{\gamma_{2} m} \\
= & -K_{1} e_{1}^{2} \\
& +\frac{\left(f_{1}-\bar{f}_{1}\right) \dot{f}_{1}}{\gamma_{1}}+\frac{\left(\gamma_{1} e_{1}+\dot{\hat{f}}_{1}\right)\left(\hat{f}_{1}-f_{1}\right)+\left(\bar{f}_{1}-\widehat{f}_{1}\right) \dot{\hat{f}}_{1}}{\gamma_{1}} \\
& +\frac{\left(\dot{\bar{m}}-e_{1} \gamma_{2} u_{1}\right)(\widehat{m}-m)+(\bar{m}-\widehat{m}) \dot{\vec{m}}}{\gamma_{2} m} .
\end{aligned}
$$

If $f_{1 \text { min }} \leq \bar{f}_{1} \leq f_{1 \text { max }}$, the following holds

$$
\widehat{f}_{1}=\bar{f}_{1}, \quad \dot{\bar{f}}_{1}=\gamma_{1} e_{1} \text {. }
$$

And if $\bar{f}_{1}<f_{1 \min }$ or $\bar{f}_{1}>f_{1 \max }$, we have

$$
\begin{aligned}
\dot{\hat{f}}_{1} & =0 \\
\left(\gamma_{1} e_{1}+\dot{\bar{f}}_{1}\right)\left(\widehat{f}_{1}-f_{1}\right) & =-\kappa_{1}\left(\bar{f}_{1}-\widehat{f}_{1}\right)\left(\widehat{f}_{1}-f_{1}\right) \leq 0,
\end{aligned}
$$

which means the following always holds:

$$
\frac{\left(\gamma_{1} e_{1}+\dot{\hat{f}}_{1}\right)\left(\hat{f}_{1}-f_{1}\right)+\left(\bar{f}_{1}-\widehat{f}_{1}\right) \dot{\hat{f}}_{1}}{\gamma_{1}} \leq 0 .
$$

Similarly, one has

$$
\frac{\left(\dot{\bar{m}}-e_{1} \gamma_{2} u_{1}\right)(\widehat{m}-m)+(\bar{m}-\widehat{m}) \dot{\bar{m}}}{\gamma_{2} m} \leq 0,
$$

so (B.2) can be rewritten as

$$
\dot{V}_{1} \leq-K_{1} e_{1}^{2}+\frac{\left(f_{1}-\widehat{f}_{1}\right) \dot{f}_{1}}{\gamma_{1}} .
$$

Based on (20), one can see that if $\widehat{f}_{1}$ tends to move out of its boundary $\left[f_{1 \text { min }}, f_{1 \text { max }}\right]$, the feedback term $-\kappa_{1}\left(\bar{f}_{1}-\widehat{f}_{1}\right)$ will pull $\bar{f}_{1}$ back to close to $\widehat{f}_{1}$; thus $\bar{f}_{1}$ is also bounded. Thus, the modified control law given by (11) and (12) can also make $e_{1}$ arbitrarily small if sufficiently large $\gamma_{1}$ and $K_{1}$ are taken.

\section{Conflict of Interests}

The authors declare that there is no conflict of interests regarding the publication of this paper. 


\section{Acknowledgments}

This paper was supported by National Science Foundation of China (51105074, 51205058, and 51375086), Foundation of State Key Laboratory of Mechanical Transmission (SKLMTKFKT-201206), and by the Polish-Norwegian Research Programme operated by the National Centre for Research and 24 Development under the Norwegian Financial Mechanism 2009-2014 in the frame of Project Contract no. PolNor/200957/47/2013.

\section{References}

[1] C. C. Chan, "The state of the art of electric, hybrid, and fuel cell vehicles," Proceedings of the IEEE, vol. 95, no. 4, pp. 704-718, 2007.

[2] J. Yamakawa, A. Kojima, and K. Watanabe, "A method of torque control for independent wheel drive vehicles on rough terrain," Journal of Terramechanics, vol. 44, no. 5, pp. 371-381, 2007.

[3] M. Shino and M. Nagai, "Independent wheel torque control of small-scale electric vehicle for handling and stability improvement," JSAE Review, vol. 24, no. 4, pp. 449-456, 2003.

[4] R. Wang and J. Wang, "Fault-tolerant control for electric ground vehicles with independently-actuated in-wheel motors," Journal of Dynamic Systems, Measurement and Control, vol. 134, no. 2, Article ID 021014, 2012.

[5] H. E. Tseng, B. Ashrafi, D. Madau, T. A. Brown, and D. Recker, "The development of vehicle stability control at Ford," IEEE/ASME Transactions on Mechatronics, vol. 4, no. 3, pp. 223234, 1999.

[6] M. Canale, L. Fagiano, and V. Razza, "Vehicle lateral stability control via approximated NMPC: real-time implementation and software-in-the-loop test," in Proceedings of the 48th IEEE Conference on Decision and Control held jointly with 2009 28th Chinese Control Conference (CDC/CCC '09), pp. 4596-4601, December 2009.

[7] M. Canale, L. Fagiano, M. Milanese, and P. Borodani, "Robust vehicle yaw control using an active differential and IMC techniques," Control Engineering Practice, vol. 15, no. 8, pp. 923941, 2007.

[8] S. Sakai, H. Sado, and Y. Hori, "Motion control in an electric vehicle with four independently driven in-wheel motors," IEEE/ASME Transactions on Mechatronics, vol. 4, no. 1, pp. 9$16,1999$.

[9] N. Mutoh, Y. Hayano, H. Yahagi, and K. Takita, "Electric braking control methods for electric vehicles with independently driven front and rear wheels," IEEE Transactions on Industrial Electronics, vol. 54, no. 2, pp. 1168-1176, 2007.

[10] D. Kim, S. Hwang, and H. Kim, "Vehicle stability enhancement of four-wheel-drive hybrid electric vehicle using rear motor control," IEEE Transactions on Vehicular Technology, vol. 57, no. 2, pp. 727-735, 2008.

[11] H. Zhang, Y. Shi, and J. Wang, "Observer-based tracking controller design for networked predictive control systems with uncertain Markov delays," International Journal of Control, vol. 86, no. 10, pp. 1824-1836, 2013.

[12] R. Wang, H. Zhang, and J. Wang, "Linear parameter-varying controller design for four wheel independently-actuated electric ground vehicles with active steering systems," IEEE Transactions on Control Systems Technology, 2013.
[13] H. Zhang, Y. Shi, and J. Wang, "Observer-based tracking controller design for networked predictive control systems with uncertain Markov delays," International Journal of Control, vol. 86, no. 10, pp. 1824-1836, 2013.

[14] H. Zhang, Y. Shi, and A. Saadat Mehr, "Robust static output feedback control and remote PID design for networked motor systems," IEEE Transactions on Industrial Electronics, vol. 58, no. 12, pp. 5396-5405, 2011.

[15] J. Wang and R. G. Longoria, "Coordinated and reconfigurable vehicle dynamics control," IEEE Transactions on Control Systems Technology, vol. 17, no. 3, pp. 723-732, 2009.

[16] J. Tjoønnäs and T. A. Johansen, "Stabilization of automotive vehicles using active steering and adaptive brake control allocation," IEEE Transactions on Control Systems Technology, vol. 18, no. 3, pp. 545-558, 2010.

[17] Z. Shuai, H. Zhang, J. Wang, J. Li, and M. Ouyang, "Combined AFS and DYC control of four-wheel-independent-drive electric vehicles over CAN network with time-varying delays," IEEE Transactions on Vehicular Technology, 2013.

[18] H. Zhou and Z. Liu, "Vehicle yaw stability-control system design based on sliding mode and backstepping control approach," IEEE Transactions on Vehicular Technology, vol. 59, no. 7, pp. 3674-3678, 2010.

[19] S. Di Cairano and H. E. Tseng, "Driver-assist steering by active front steering and differential braking: design, implementation and experimental evaluation of a switched model predictive control approach," in Proceedings of the 49th IEEE Conference on Decision and Control (CDC '10), pp. 2886-2891, December 2010.

[20] J. Wang, L. Alexander, and R. Rajamani, "Friction estimation on highway vehicles using longitudinal measurements," Journal of Dynamic Systems, Measurement and Control, vol. 126, no. 2, pp. 265-275, 2004.

[21] R. Wang, Y. Chen, D. Feng, X. Huang, and J. Wang, "Development and performance characterization of an electric ground vehicle with independently actuated in-wheel motors," Journal of Power Sources, vol. 196, no. 8, pp. 3962-3971, 2011.

[22] S. Horiuchi, K. Okada, and S. Nohtomi, "Improvement of vehicle handling by nonlinear integrated control of four wheel steering and four wheel torque," JSAE Review, vol. 20, no. 4, pp. 459-464, 1999.

[23] J. Y. Wong, Theory of Ground Vehicles, Wiley, New York, NY, USA, 3rd edition, 2001. 


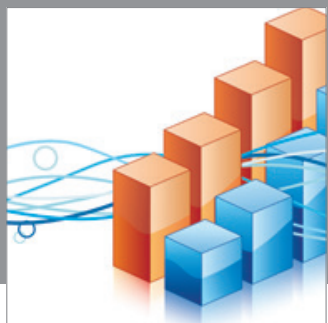

Advances in

Operations Research

mansans

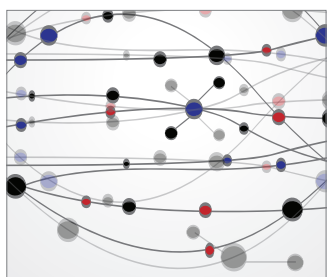

The Scientific World Journal
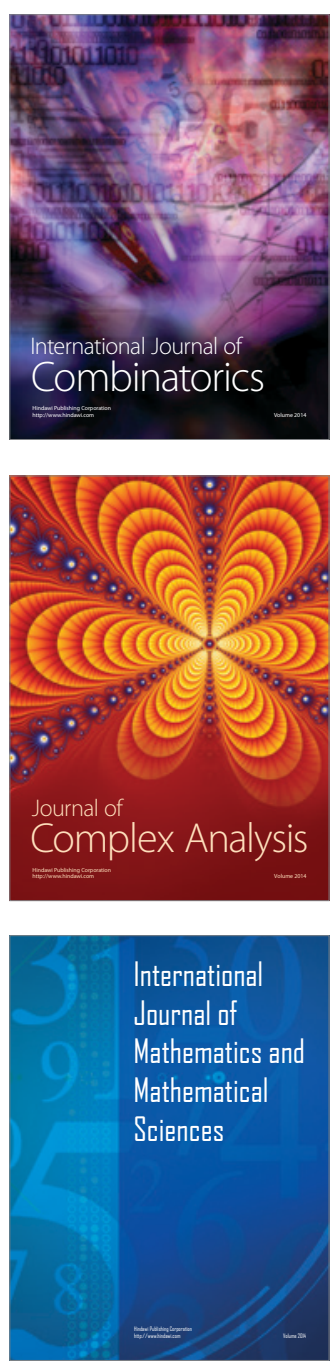
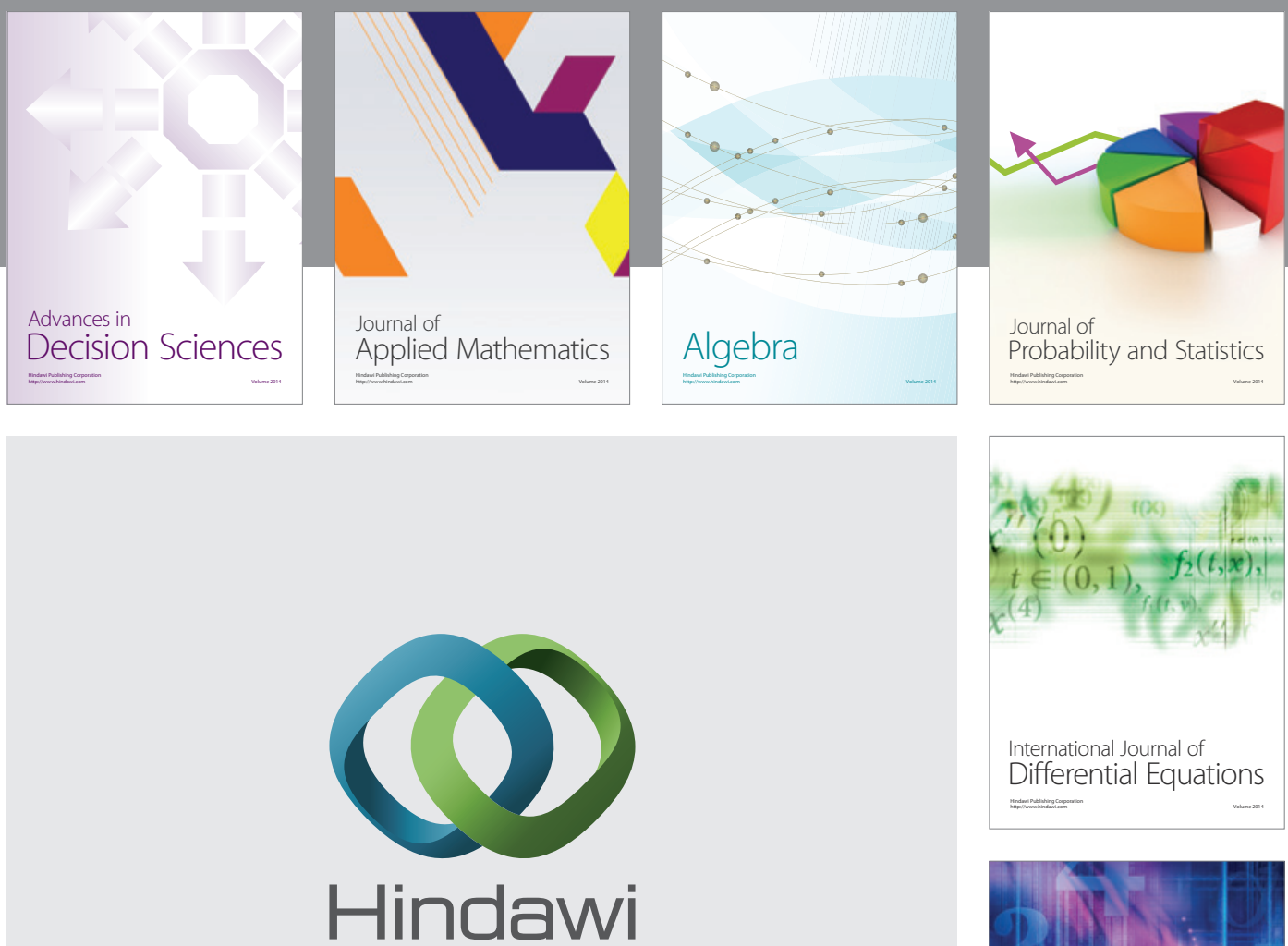

Submit your manuscripts at http://www.hindawi.com
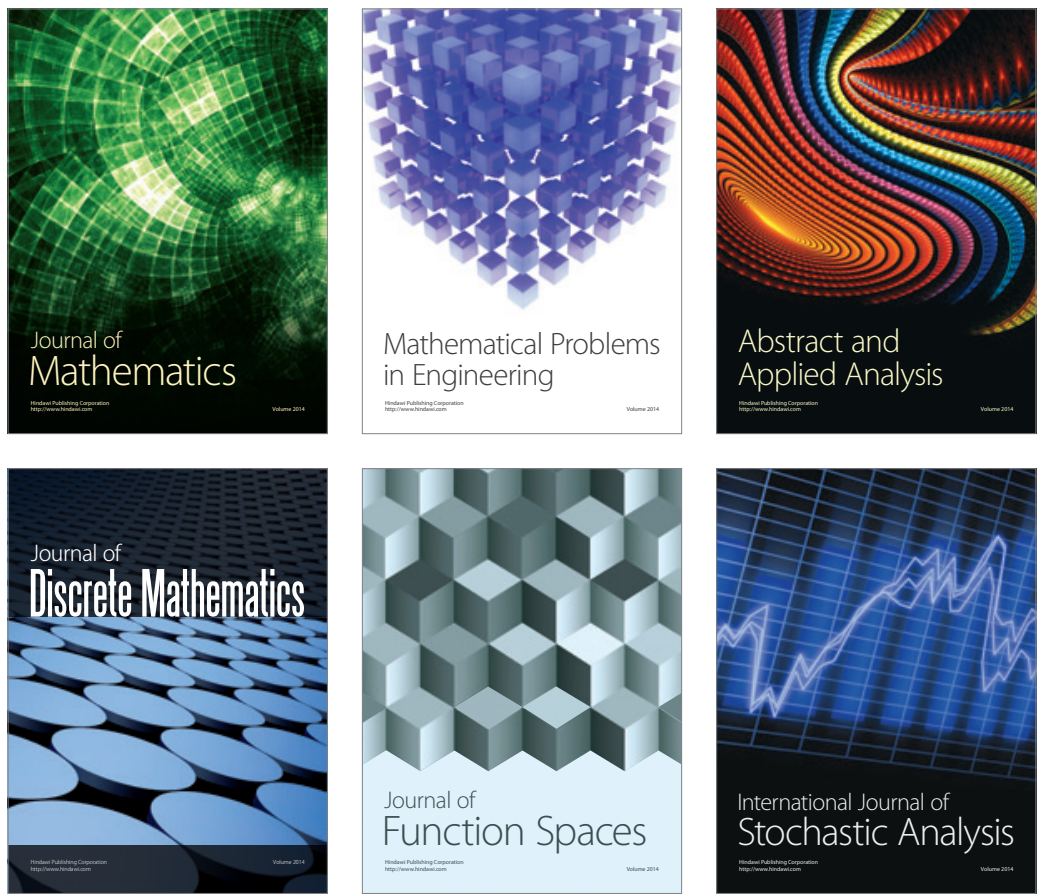

Journal of

Function Spaces

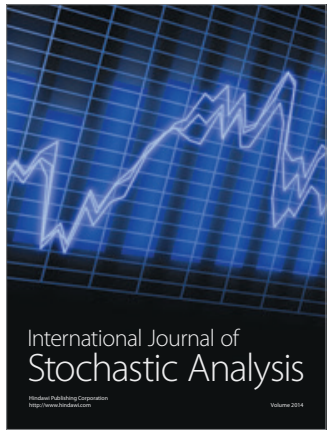

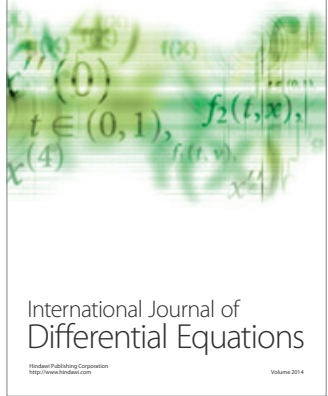
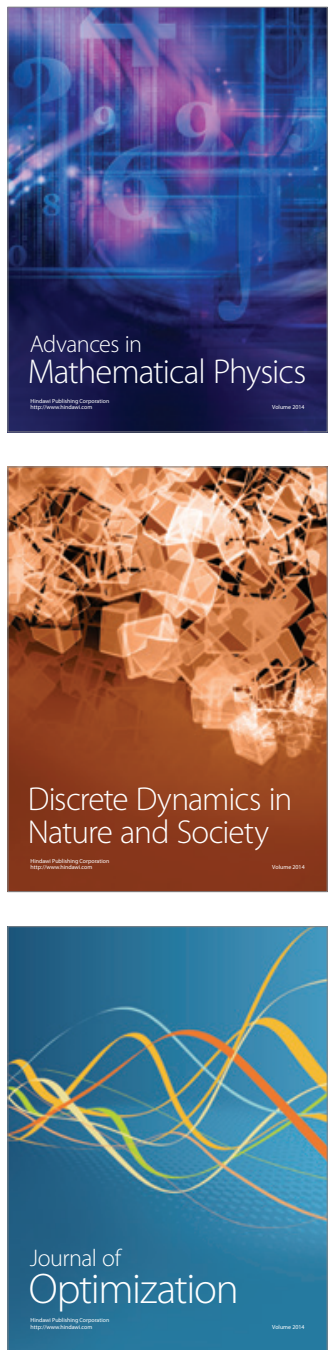